\title{
Multiple Sclerosis and Repeat Dislocations of Total Knee Replacements: A Case Report
}

\author{
Masood Rehman Moghula, b, Bassel El-Osta ${ }^{a}$, Andrew Osborne ${ }^{a}$, John Hollingdale ${ }^{\mathrm{a}}$
}

\begin{abstract}
We present a case report of a 63-year-old patient with known multiple sclerosis (MS) undergoing a knee replacement with a Birmingham prosthesis. Eight days following successful joint replacement rehabilitation remained slow. The patient was found to have an innocuous posterior dislocation of the prosthesis with no apparent explanation. He underwent open reduction of the joint and a cricket-pad splint was applied. Forty-eight hours later the patient had a repeat posterior dislocation, again with no explanation. Finally a cast was applied and later the patient discharged to a rehabilitation facility. Four-months after discharge the patient remained well with the prosthesis in situ, although unable to weight bear. We advise considerable thought taken before recommending patients with MS for joint replacement.
\end{abstract}

Keywords: Knee replacement; Dislocation; Multiple sclerosis

\section{Introduction}

Dislocations involving knee prostheses have been documented extensively. Rates in fixed bearing implants vary between $0.15-0.5 \%$. However, this is only the third reported case in a patient with multiple sclerosis (MS), and the first such case where the patient did not report any spasms. As such we believe it to be a valuable report indicating that careful consideration needs to be taken before proceeding

Manuscript accepted for publication October 21, 2011

${ }^{a}$ North-West London Hospitals NHS Trust, UK

${ }^{\mathrm{b} C o r r e s p o n d i n g ~ a u t h o r: ~ M a s o o d ~ R e h m a n ~ M o g h u l, ~}$

Email: masood.moghul@doctors.org.uk

doi:10.4021/jmc391w with joint replacement surgery.

\section{Case Report}

A 63-year-old man presented with severe tricompartmental primary arthritis of the right knee shown on both athroscopy and radiographs (Fig. 1). He was known to the orthopaedic team for some time having had a previous osteotomy in the right knee for realignment due to a valgus deformity, in the 1980s.

He had suffered from symptomatic secondary progressive MS since 1970 with lower limb weakness, particularly in his quadriceps. Pre-operatively his left leg was affected more by the MS than the right hence it was decided to operate with prior consultation from his neurologists. He had been mobilising with a Zimmer frame and a wheelchair for longer distances. He was using Baclofen but this was replaced by Pregabalin two months prior to surgery, due to nausea.

The right knee was replaced, by the senior author, (firstly removing the 3 staples) using a Birmingham prosthesis (Fig. 2 ). The posterior cruciate ligament was retained in line with his standard practice. Medium tibial and large femur prostheses were used with a $12.5 \mathrm{~mm}$ insert and $5 \mathrm{~mm}$ patella offset. The procedure lasted approximately 2 hours. Post-operatively the patient had urinary retention which was a recurring issue for him. Full weight bearing mobilisation was attempted post-operatively however this was impeded by muscle spasms and generalised muscle weakness, with right knee flexion at $30^{\circ}-40^{\circ}$. Pregabalin was continued to aid his mobility prior to his physiotherapy sessions.

Eight days post-operatively the patient's mobility remained very poor, barely being able to stand with the assistance of the physiotherapy team. During a routine ward round a deformity of the knee replacement was observed. A repeat X-ray revealed a posterior dislocation (Fig. 3). No obvious explanation was found and there was no evidence of any trauma. The patient was taken to theatre and a closed reduction was attempted. This was unsuccessful and an open exploration of this was performed, taking approximately 30 minutes. The implants were seen to be stable without any damage, the knee fully extended and flexed to $110^{\circ}$. 


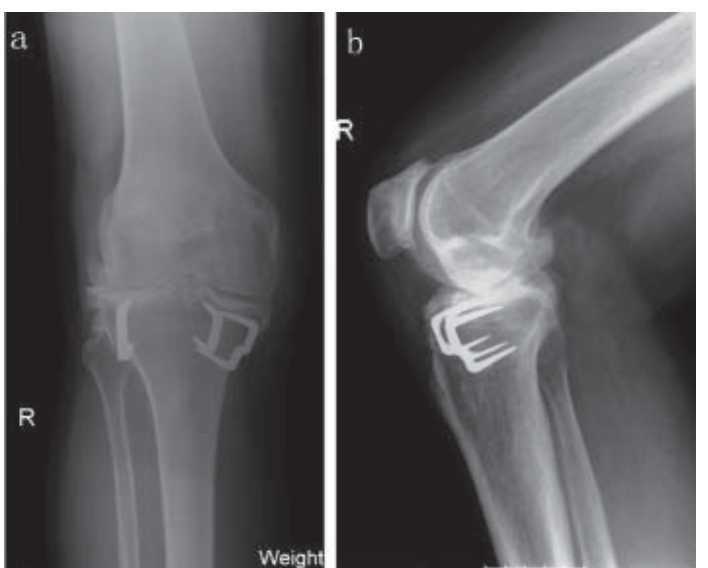

Figure 1. Pre-operative AP: $a$, and lateral: $b$ of $X$-rays of the right knee (weight bearing)
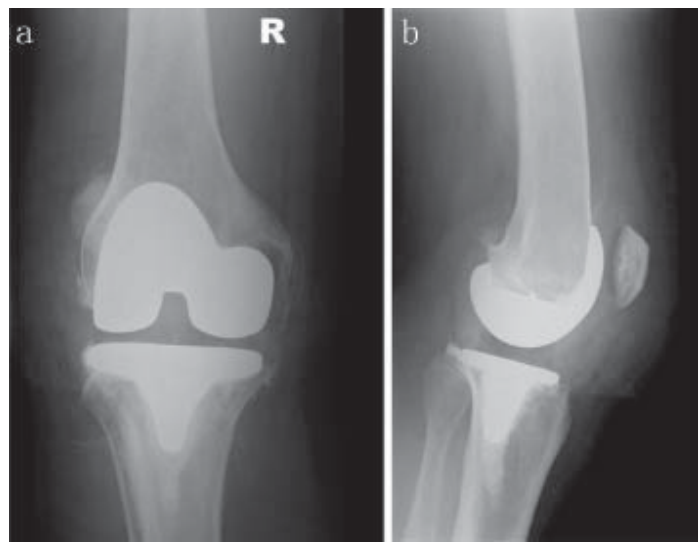

Figure 2. Right knee X-rays post-arthroplasty (AP: a, and lateral: b)

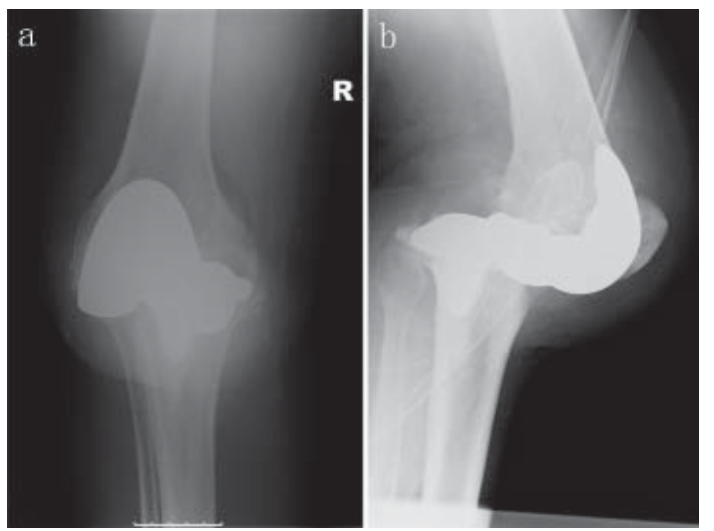

Figure 3. Dislocated right knee X-rays (AP: $a$, and lateral: b)

Following successful reduction the patient was taken back to the ward with a cricket pad splint, to be worn at all times.

Forty-eight hours after reduction a repeat X-ray showed the patient had dislocated posteriorly again, despite all previous management (Fig. 4). Once more there was no

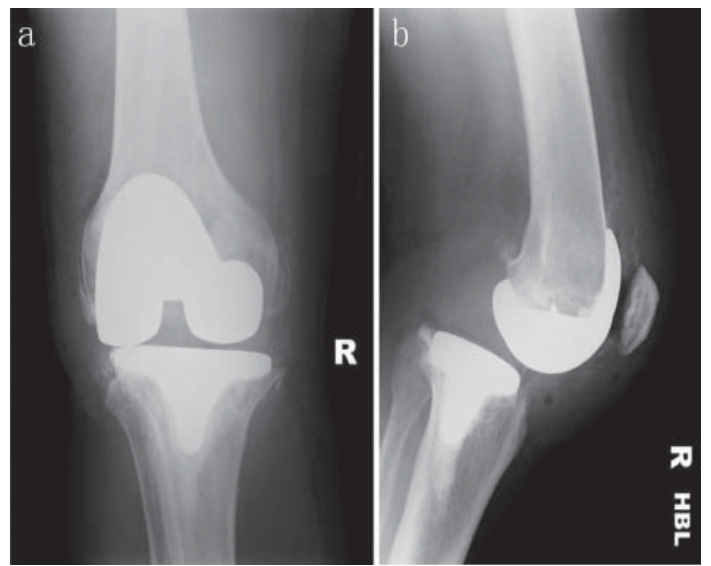

Figure 4. Second dislocation X-rays (AP: $a$, and lateral: $b$ )

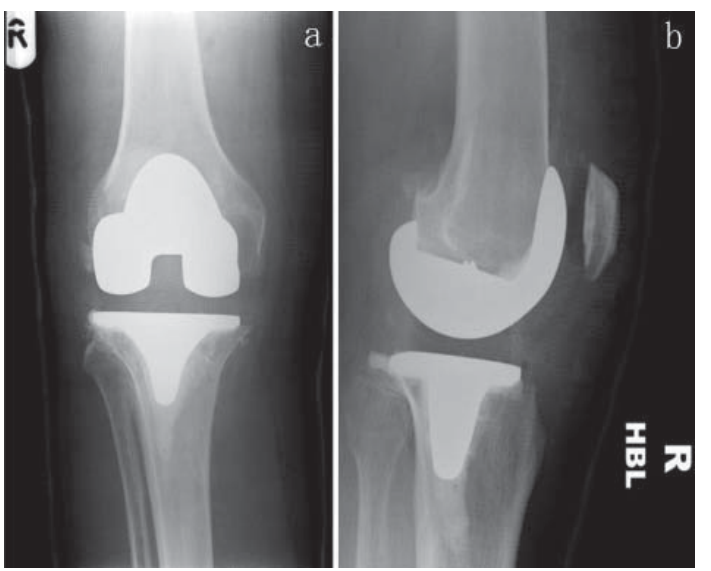

Figure 5. Right knee relocated and in plaster (AP: a, and lateral: b)

history of trauma and the cause was unclear. He underwent a further manipulation under anaesthesia, but closed, and application of Plaster of Paris. Repeat X-ray showed the prosthesis in place (Fig. 5). The patient denied feeling any spasms throughout.

Nine days after his second reduction the patient had managed to stand successfully with a maximum assistance of 2. At this point he was medically stable and discharged to a rehabilitation facility to continue his rehab, with his knee still in Plaster of Paris and his MS well controlled with no spasms.

At 4-month follow-up his prosthesis was in situ (Fig. 6) although the patient was unable to weight bear.

\section{Discussion}

Dislocations involving knee prostheses have been reported previously [1-4], with rates in fixed bearing implants of $0.15-0.5 \%$ [4]. Although various associations have been 


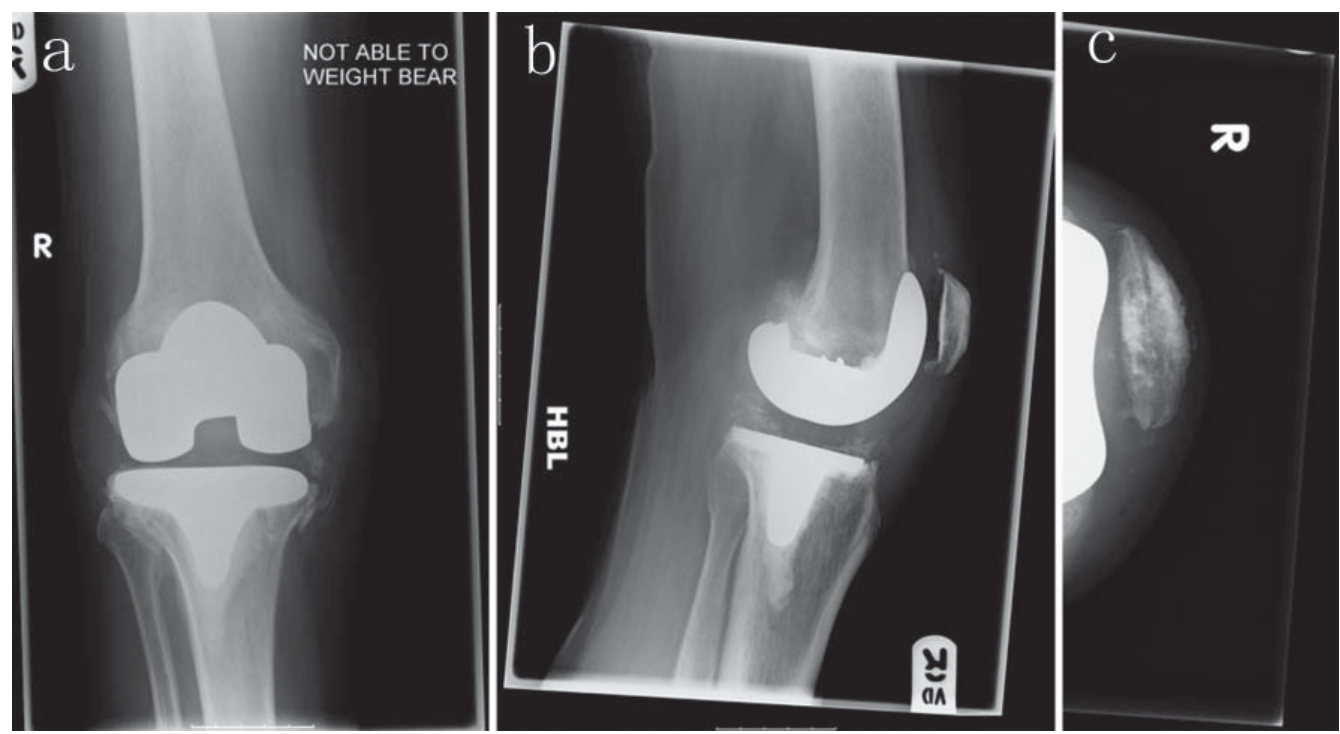

Figure 6. Four-month follow-up of right knee (AP: a, lateral: b, and skyline: c)

noted, varus/valgus deformity, ligament laxity [1], and several simple post-operative measures advised to prevent dislocation [5]. However, to our knowledge this is only the third reported case with the patient suffering from MS, and the first such case where the patient did not report any spasms. Hence we advise that caution should be taken when contemplating joint prostheses in patients with MS, even if well controlled.

\section{References}

1. Gebhard JS, Kilgus DJ. Dislocation of a posterior stabilized total knee prosthesis. A report of two cases. Clin Orthop Relat Res. 1990;254:225-229.
2. Erceg M, Maricevic A. Recurrent posterior dislocation following primary posterior-stabilized total knee arthroplasty. Croat Med J. 2000;41(2):207-209.

3. Dawson-Bowling S, Tavakkolizadeh A, Cottam HL, Butler-Manuel PA. Multiple sclerosis and bilateral dislocations of total knee replacements: a case report. Knee Surg Sports Traumatol Arthrosc. 2008;16(2):148-151.

4. Rao V, Targett JP. Instability after total knee replacement with a mobile-bearing prosthesis in a patient with multiple sclerosis. J Bone Joint Surg Br. 2003;85(5):731732.

5. Lombardi AV, Jr., Mallory TH, Vaughn BK, Krugel R, Honkala TK, Sorscher M, Kolczun M. Dislocation following primary posterior-stabilized total knee arthroplasty. J Arthroplasty. 1993;8(6):633-639. 